\title{
A EDUCAÇÃO A DISTÂNCIA NO CONTEXTO DA POLÍTICA NACIONAL DE SAÚDE DA PESSOA IDOSA: ESTUDO DE EGRESSOS
}

\author{
DISTANT LEARNING IN THE CONTEXT OF THE BRAZILIAN NATIONAL \\ ELDERLY HEALTH POLICY: A COURSE EGRESSED STUDY
}

\author{
EDUCACIÓN A DISTANCIA EN EL CONTEXTO DE LA POLÍTICA NACIONAL DE \\ SALUD PARA LA VEJEZ: UN ESTÚDIO DE PROFESIONALES EGRESOS
}

\author{
Kellem Raquel Brandão de Oliveira Torres ${ }^{1}$ \\ Vera Lucia Luiza ${ }^{2}$ \\ Mônica Rodrigues Campos
}

Resumo A pesquisa que deu origem a este artigo teve como objetivo analisar a efetividade de um curso sobre envelhecimento na implementação da Política Nacional de Saúde da Pessoa Idosa com base na percepção dos profissionais de saúde egressos. Aplicou-se questionário eletrônico autorrespondido por 414 de 1.561 egressos da primeira à terceira ofertas do curso, de julho a outubro de 2015. A variável-desfecho para medir a efetividade do curso foi a declaração de melhora no desempenho dos profissionais egressos em pelo menos cinco de dez atividades relacionadas à saúde do idoso previstas na referida política. Houve melhora na realização de todas essas atividades, com variação de 1,3 a 2,6 vezes mais quanto ao pré e ao pós-curso. Formação profissional, tempo de trabalho com saúde do idoso e implementação prática do aprendizado obtido no curso foram os aspectos que explicaram de modo mais justo a melhora nas atividades da política (via modelo de regressão logística multivariada). O estudo procurou reforçar a importância da educação permanente como ferramenta para formação/capacitação dos profissionais que atuam no Sistema Único de Saúde, tendo se mostrado uma efetiva estratégia para implementação de atividades da mencionada política destinada à pessoa idosa.

Palavras-chave saúde do idoso; educação a distância; educação permanente.
Abstract This article aims to analyse the effectiveness of a course in aging and health of elderly people as part of the implementation of the Brazilian National Elderly Health Policy (PNSPI), from the perception of health professionals egressed from the course. A self-responded electronic questionnaire was applied from July to October 2015, answered by 414 in 1,561 egresses. The outcome variable to measure the course effectiveness was the improvement in the performance of egresses in at least five in ten activities related to health of elderly as provided for in PNSPI. There was improvement in all PNPSI activities varying between 1.3 to 2.6 times pre and post course. Professional background, time working with elderly health and actual implementation of learning from the course were the aspects that explained better the improvement in PNSPI activities (trough logistic regression model). The course proved have been effective for the implementation of PNSPI activities, showing the potential of continuing education to enforce public policies.

Keywords health of the elderly; distance education; permanent education. 


\section{Introdução}

O aumento progressivo da população idosa, associado a fatores como o crescimento da expectativa de vida, a queda da taxa de fecundidade e a redução da mortalidade em todos os grupos etários - processo conhecido como transição demográfica e epidemiológica -, vem alterando a pirâmide populacional de vários países em todo o mundo. Apenas na virada do século XX, porém, esse crescimento na expectativa de vida se traduziu em mudanças relevantes na demografia dos países e tornou-se grande desafio para a saúde pública e para os sistemas de saúde mundiais, no que se refere a proporcionar um atendimento adequado à população (Silvestre e Costa Neto, 2003; Veras e Caldas, 2004).

O envelhecimento implica necessidades próprias de saúde, com o aumento da frequência e a gravidade de problemas, sobretudo os crônicos, que perduram por toda a vida do indivíduo, além das deficiências próprias da senescência. Ademais, é uma população que tende a perder a autonomia de seu cuidado. Estudos populacionais realizados no Brasil têm demonstrado que pelo menos $85 \%$ dos idosos apresentam ao menos uma doença crônica, e cerca de $10 \%$ apresentam pelo menos cinco dessas enfermidades (Brasil, 2002; Silvestre e Costa Neto, 2003).

No conjunto das capitais dos 26 estados brasileiros e no Distrito Federal, a frequência de diagnóstico médico prévio de hipertensão arterial foi de $24,8 \%$, sendo maior em mulheres $(26,8 \%)$ do que em homens $(22,5 \%)$. Para o diabetes, essa frequência foi de $8 \%$, com 7,3\% e 8,7\% em homens e mulheres, respectivamente. A frequência de diagnósticos aumentou muito com a idade e a escolaridade, em ambos os sexos, em particular depois dos 45 anos. Aproximadamente um quarto dos indivíduos com 65 ou mais anos de idade $(24,4 \%)$ referiram diagnóstico médico de diabetes (Malta et al., 2015; Brasil, 2015).

Em estudo realizado em 2016, 7,6\% da população tinha 65 anos ou mais no Brasil. Essa parcela será de 9,4\% em 2020 e 11,3\% em 2025, padrão similar ao de alguns países ricos (Gomes, 2016). Tal mudança vai requerer significativo aumento da oferta de infraestrutura de saúde, já que a população idosa usa muito mais esses serviços.

O Sistema Único de Saúde (SUS) visa proporcionar ao cidadão um atendimento mais humanizado, maximizando o acesso e priorizando as ações de prevenção, promoção e recuperação de forma integral e continuada. Para isso, o Ministério da Saúde (MS) vem desenvolvendo programas e reformas institucionais com o objetivo de fortalecer cada vez mais o SUS, como o Pacto pela Saúde, expresso em 'três' dimensões: Pacto pela Vida, Pacto de Gestão e Pacto em Defesa do SUS (Brasil, 1997).

O Pacto pela Vida traz a atenção à saúde do idoso no topo da lista de objetivos e, como meta prioritária, a implantação da Política Nacional de Saúde da Pessoa Idosa (PNSPI), buscando a atenção integral. Reforça no SUS o movimento da gestão pública por resultados, estabelece um conjunto de compromissos sani- 
tários considerados prioritários, pactuado e implementado de forma tripartite. Esses compromissos deverão ser efetivados pela rede do SUS, de forma a garantir o alcance das metas estabelecidas. A avaliação é um componente importante do processo das políticas públicas, gerando evidências para que se conheça o alcance de seus objetivos, além de permitir compartilhar informações com os atores envolvidos, formuladores, profissionais e usuários (Hartz, 2013).

A Estratégia Saúde da Família (ESF), importante modelo de assistência, ordenador da atenção primária à saúde no Brasil, vem revelando a presença de idosos e famílias frágeis e em situação de grande vulnerabilidade social por meio do processo de cadastramento das famílias na delimitação do território de abrangência das equipes. A ESF representa não só para o idoso, mas para toda a população, o vínculo com o sistema de saúde (Silvestre e Costa Neto, 2003).

No nível dos estados, uma importante estratégia são as redes estaduais de assistência à saúde do idoso, que visam assegurar o atendimento às necessidades e promover o envelhecimento saudável - de forma que o idoso mantenha sua capacidade funcional, promovendo assim redução no número e no tempo das internações hospitalares (Brasil, 2002).

A vigência do Estatuto do Idoso, a ampliação da ESF, as redes estaduais de assistência à saúde do idoso e a publicação do Pacto pela Saúde tornaram imperiosa a readequação da PNSPI (Brasil, 2006). Uma de suas diretrizes é a "formação e educação permanente dos profissionais do SUS, na área de saúde da pessoa idosa" (Brasil, 2006, p. 6).

No cenário econômico atual, globalizado e produtivo, o uso de novas tecnologias agregadas à produção e aos serviços modifica-se o tempo todo; por isso, devese buscar o conhecimento por meio da educação permanente (Rodrigues, 1994).

Na América Latina, os primeiros debates sobre educação permanente voltada para capacitação de trabalhadores da saúde são de 1985 (Lima et al., 2010). No Brasil, os desafios trazidos pela implantação do SUS, a operacionalização do conceito de saúde integral e a adoção da ESF impuseram a necessidade do desenvolvimento de diversas formas de capacitação para os profissionais atuantes nessa área. Assim, a utilização da educação permanente no campo da saúde justifica-se pela necessidade de produzir profissionais capazes de proporcionar aos usuários do SUS uma atenção que fortaleça todos os princípios pactuados.

O MS criou, em 2003, a Secretaria de Gestão do Trabalho e da Educação na Saúde e instituiu, em 2004, a Política Nacional de Educação Permanente em Saúde (PNEPS), na qual previa a criação de polos de educação permanente em saúde em todo o país (Brasil, 2004, 2005; Lima et al., 2010). Essa iniciativa visava desencadear um processo de educação permanente em saúde (EPS) dos trabalhadores para o desenvolvimento de uma atenção integral à população e ao fortalecimento do SUS, desde a formação técnica desses profissionais até a pós-graduação. 
Em 2007, a PNEPS passou por um processo de redefinição de diretrizes e estratégias com o intuito de se adequar ao Pacto pela Saúde. A portaria resultante desse processo (Brasil, 2007) extinguiu os polos de EPS e criou as comissões permanentes de integração ensino-serviço (CIESs) - instâncias previstas no Pacto pela Saúde em substituição aos polos de EPS. As CIESs, juntamente com os colegiados de gestão regional, assumiram a responsabilidade pela elaboração do Plano de Ação Regional de Educação Permanente em Saúde, bem como pelo desenvolvimento da política de EPS no espaço de cada região.

A EPS é considerada pelo MS como "uma ação finalística da política de saúde e não mais como atividade-meio para o desenvolvimento da qualidade do trabalho" (Ceccim, 2005, p. 975). A EPS ultrapassa os limites da transmissão de conhecimentos definitivos, de conceitos acabados. Aplicada ao campo da saúde pública, faz-se essencial para acompanhar esse campo do saber que está em constante construção e evolução (Gadotti, 1992).

Além de uma opção didático-pedagógica, a EPS expressa a articulação entre o ensino, o trabalho e a cidadania, tendo a integralidade na condição de diretriz do cuidado à saúde e a participação popular como papel de controle social. Dessa forma, a educação permanente em saúde precisa ser entendida, ao mesmo tempo, como uma "prática de ensino-aprendizagem" e como uma "política de educação na saúde" (Ceccim e Ferla, 2009).

Até pouco tempo, as relações entre professores e alunos dependiam da presença física de ambos em determinado espaço. Hoje, as novas modalidades de ensino, principalmente as que se utilizam das novas tecnologias, já possibilitam ao aluno participar de cursos de diversos níveis, sem que para isso seja necessário estar no mesmo espaço físico que o professor. A chamada educação a distância (EAD), apontada como alternativa importante para a oferta de cursos, é considerada maneira viável e eficiente de promover formação simultânea para grande quantidade de pessoas no menor tempo possível (Bello e Rodrigues, 2012).

A modalidade de EAD representa para os profissionais da saúde, sobretudo num país de dimensões continentais como o Brasil, uma alternativa viável para a formação permanente, na medida em que eles não precisam se afastar de seu local de trabalho para estudar. É um fator de grande relevância para essa clientela, com múltiplos vínculos de trabalho (Leitão et al., 2000).

Nesse cenário, a Escola Nacional de Saúde Pública Sergio Arouca (Ensp), uma das unidades da Fundação Oswaldo Cruz (Fiocruz), destaca-se com a oferta de cursos presenciais e a distância em diversos níveis de ensino. Sediada no campus da Fiocruz, em Manguinhos, no Rio de Janeiro, a Ensp atua na capacitação e formação de alunos, na produção científica e tecnológica e na prestação de serviços à saúde pública; mantém programas de cooperação técnica com todos os estados do Brasil e com instituições nacionais e internacionais, atuantes no campo da saúde. Os referenciais político-pedagógicos assumidos pela EAD/ Ensp sustentam-se na compreensão de que educação a distância é, antes de tudo, 
educação, a qual está associada ao contexto histórico-social, do qual o trabalho humano é constituinte (Coimbra et al., 2008).

O objetivo do processo educacional é desenvolver competências e habilidades. Competência é aqui entendida como a faculdade de mobilizar um conjunto de recursos cognitivos (saberes, capacidades, informações etc.) para solucionar com pertinência e eficácia uma série de situações (Perrenoud, 2000). As habilidades são o saber-fazer relacionado com a prática do trabalho, transcendendo a mera ação motora. Ou seja, as habilidades são atributos relacionados não apenas ao saber-fazer, mas aos saberes (conhecimentos), ao saber-ser (atitudes) e ao saberagir (práticas no trabalho).

O sistema de ensino superior se constitui sobre uma base multidimensional para atendimento de diversas necessidades de transformação dos diversos atores da sociedade. O que o governo, o setor acadêmico, a sociedade e os setores produtivos demandam das instituições de ensino superior pode ser distinto entre eles. Assim, o sentido da qualidade da educação superior deve caminhar para o consenso satisfatório entre todas essas questões, buscando adaptar essa perspectiva da multidimensionalidade por meio de avaliações que sejam propostas para a integração dos diversos atores desse processo (Abreu Jr., 2009). Ao considerar o ex-aluno (egresso) no processo de avaliação institucional, tenta-se mensurar e promover ações para atender satisfatoriamente a diversidade de interesses sob os quais as universidades se encontram hoje.

É de extrema relevância identificar os fatores que são de importância para os clientes e compará-los com o desempenho apresentado pela organização, para que seja possível tomar as decisões adequadas, permitindo aproximar esses critérios do cliente ao desempenho da organização (Brochado, Phiton e Pereira, 2006; Cordeiro e Motta, 2008). Desse modo, "os discentes como principais clientes do serviço prestado pela instituição podem ser a referência para atestar essa qualidade e, por consequência, contribuir para a continuidade desse serviço" (Cordeiro e Motta, 2008, p. 4).

$\mathrm{O}$ egresso de um curso tem absoluta centralidade no processo formativo, no qual assume distintos papéis. É o cliente, por participar do processo educacional na expectativa de uma posição no mercado advinda de sua qualificação. É o aluno que está sendo transformado e, nesse sentido, a entrada do processo (Brochado, Phiton e Pereira, 2006), mas é igualmente resultado do processo de formação. Também podemos pensar assim a respeito dos egressos da pós-graduação, pois é pelo curso que adquirem mais conhecimento voltado para uma área específica e podem aplicar na realidade prática, transformando-a.

É muito importante a instituição receber esse retorno da qualidade dos formandos que estão sendo oferecidos à sociedade quanto à aptidão para a atividade profissional. Isso porque as mudanças drásticas que vêm ocorrendo no mundo do trabalho, da economia e da sociedade - como a globalização, os avanços tecnológicos, a crescente oferta de cursos superiores e as novas exigências 
de preparação dos profissionais - obrigam as instituições de ensino superior a formarem profissionais cada vez mais multidisciplinares, capazes de exercerem diferentes funções, realizarem diferentes atividades, resolverem problemas com rapidez e segurança, além daqueles ligados à sua área de formação (Lousada e Martins, 2005).

Outro motivo para a inserção da perspectiva do egresso na avaliação do ensino superior é o fato de que as tendências e exigências profissionais estão em constante transformação, e os egressos poderão expressar em que medida a proposta pedagógica os preparou para confrontar tais exigências.

Entretanto, essa inserção não deve ocorrer apenas com confecção de pesquisas isoladas, e sim realizando o acompanhamento sistemático desses egressos por pelo menos um período de tempo predeterminado, para que se possibilite a análise da evolução da trajetória profissional, do impacto do curso no seu desempenho profissional e dos resultados desse processo para a sociedade (Espartel, 2009).

O curso de aperfeiçoamento em envelhecimento e saúde da pessoa idosa (Caespi) é oferecido pela Ensp na modalidade EAD desde 2008. Tem natureza interdisciplinar, e seu objetivo geral é promover a qualificação de profissionais de nível superior do setor da saúde que atuam na rede básica de assistência, com ênfase na ESF. Busca disseminar e implementar princípios de uma política de governo quanto à saúde da pessoa idosa, operacionalizando atividades que visem à prevenção de perdas, à manutenção e à recuperação da capacidade funcional da população idosa e ao controle dos fatores que interferem no estado de saúde (Coimbra et al., 2008).

Os cursos realizados pela EAD/Ensp/Fiocruz são, em sua maioria, financiados pelo governo federal (MS). O planejamento da oferta dos cursos é baseado em demandas para capacitação dos profissionais dos municípios e estados nas políticas de saúde existentes. No entanto, não há estudos sobre a avaliação de egressos dos cursos da EAD/Ensp/Fiocruz no que tange a efeitos concretos na política objeto do curso. Poucos são os cursos que passam por um processo de avaliação na sua finalização para identificar se a demanda foi realmente atendida e se ainda há necessidade de novas capacitações naquela área e para qual número de profissionais.

Com relação ao curso, objeto do estudo aqui apresentado, apesar de ser uma relevante iniciativa ante a PNSPI, não existe avaliação sobre seu resultado quanto à mudança dos processos de trabalho no SUS, ou seja, se causou algum impacto na rede de atendimento ao idoso. Assim, torna-se importante identificar, nos municípios com profissionais egressos, se o curso foi capaz de favorecer a implementação da PNSPI, melhorando assim a rede de atendimento.

A pesquisa teve como objetivo geral analisar a efetividade do Caespi da EAD/Ensp/Fiocruz na implementação da PNSPI, com base na percepção dos profissionais de saúde egressos do curso. Por efetividade de uma intervenção, 
entendemos o alcance de objetivos nas condições reais de operação (Viacava et al., 2012).

O estudo fez parte da dissertação de mestrado da primeira autora, intitulada Educação a distância como estratégia para apoio à implementação de políticas públicas: a experiência do curso de aperfeiçoamento em envelhecimento e saúde da pessoa idosa (EAD/Ensp/Fiocruz), que contou com bolsa da Coordenação de Aperfeiçoamento de Pessoal de Nível Superior (Capes). Não houve financiamento específico. A pesquisa foi aprovada pelo Comitê de Ética em Pesquisa da Ensp/Fiocruz.

\section{Método}

Tratou-se de um estudo transversal, descritivo quanto ao perfil dos egressos e analítico no que tangia à avaliação da contribuição do curso na implementação da PNSPI.

\section{Obtenção dos dados}

O curso possui uma base de dados (Simios) sobre os alunos e seu processo de desenvolvimento acadêmico. A primeira oferta (quinhentas vagas) foi voltada para as regiões Norte/Nordeste nos anos de 2009/2010 (chamemos de oferta A) e a segunda e a terceira ofertas (duas mil vagas) para as regiões Sul/ Sudeste/Centro-Oeste, realizadas em 2011 e 2012/2013 (chamemos de oferta B). Da oferta A, 38,6\% se formaram; e da oferta B, 68,4\%. Era um pré-requisito para matrícula uma declaração do candidato quanto a estar atuando diretamente com população idosa, seja no atendimento, seja na gestão dos programas voltados para o idoso. Identificaram-se 1.566 alunos formados no conjunto das ofertas. Para evitar incoerências, foram excluídos cinco egressos das regiões Sudeste, Sul e Centro-Oeste que realizaram o curso na primeira oferta (voltada para as outras regiões do país). Assim, o questionário foi enviado eletronicamente para 1.561 profissionais formados $(62,4 \%)$ das três primeiras ofertas do curso (ofertas A e B). Na base Simios, obtiveram-se informações sobre sexo, idade, formação, município e estado de atuação dos egressos das ofertas em estudo.

O questionário foi composto por 19 questões, distribuídas em quatro blocos (informações sobre o egresso; informações acadêmicas; informações profissionais; e avaliação do egresso sobre a realização do curso). 


\section{Variáveis de interesse}

A variável-desfecho para medir o impacto do curso foi expressa por meio da melhora no desempenho dos profissionais egressos em pelo menos cinco de dez atividades relacionadas à saúde do idoso previstas na PNSPI ('ter melhora'), que representou cerca de $25 \%$ dos egressos, correspondendo ao terceiro quartil da variável 'número de atividades4 com melhora'.

Como variáveis de exposição, consideraram-se: sexo; idade (até 50 anos, 50 anos ou mais); porte populacional do município de atuação (até 50; 50-100 e acima de 100 mil habitantes); oferta do curso que participou (A: região Norte/ Nordeste; ou B: região Sul/Sudeste/Centro-Oeste); formação básica (assistente social/fisioterapeuta/psicólogo, enfermeiro ou médico e outras profissões); estar cursando ou ter cursado outro curso na mesma área; desenvolver a mesma função da época do curso; tempo de trabalho com saúde do idoso (até cinco anos; mais de cinco anos); satisfação com o trabalho com saúde do idoso (muito satisfeito e satisfeito, pouco ou nada satisfeito); continuar trabalhando no mesmo município da época do curso; atividade principal na saúde do idoso (assistencial/educativa ou outras atividades); achar que o curso foi fortemente relacionado ao trabalho na saúde do idoso; achar que a formação recebida no curso é compatível com a necessidade de conhecimento para a prática atual; ter conseguido implementação prática do curso; aspectos referidos como facilidade/dificuldade para implantação ou aprimoramento das atividades da saúde do idoso - gestão local de saúde, estrutura física do local, infraestrutura de apoio, acesso da população ao serviço, quantidade adequada de profissionais, quantidade suficiente de profissionais capacitados e rede de serviço de saúde.

\section{Aspectos operacionais}

As opções apresentadas à pergunta sobre aspectos que representaram facilidades ou dificuldades para a implementação das atividades relacionadas à saúde do idoso originaram-se das respostas mais mencionadas na questão aberta do instrumento de avaliação formativa dos alunos sobre o curso, preenchida ao final de cada oferta (Torres, 2012).

Após realização de teste-piloto, o convite para participar da pesquisa foi encaminhado via e-mail pelo sistema de avaliação EAD/Ensp/Fiocruz. O texto de apresentação continha informações sobre o estudo e orientações de preenchimento, seguido de um link para acesso ao questionário.

O projeto foi submetido ao Comitê de Ética da Ensp/Fiocruz (parecer n. 1.090.580) e seguiu as recomendações da resolução n. 466/12. Diante da grande autonomia permitida pela interação eletrônica, a anuência foi manifestada 
pela indicação no campo apropriado, além de o acesso à página do questionário ser considerado anuência ao termo de consentimento livre e esclarecido.

Para garantir a melhor taxa de resposta, foram encaminhados, de forma periódica, lembretes aos participantes que não haviam iniciado ou completado o preenchimento. Obtivemos 414 respostas $(26,52 \%)$, e apenas sete pessoas se posicionaram informando não desejar participar da pesquisa.

\section{Análise}

Os dados foram exportados para o SPSS 17, compondo diferentes bancos onde realizamos as análises. Em algumas etapas do trabalho, utilizamos adicionalmente o Microsoft Excel.

Depois da descrição univariada de todos os indicadores e variáveis de exposição, realizou-se análise bivariada entre variáveis de exposição e desfecho considerado: tipo de oferta (A: Norte/Nordeste; ou B: Sul/Sudeste/ Centro-Oeste) e 'ter melhora' a partir do curso.

Para a verificação da existência de associação entre as características do perfil e as ofertas do curso, aplicaram-se os testes de qui-quadrado de associação (Pearson) e, quando pertinente, o exato de Fischer, com nível de significância de $5 \%$.

De modo a medir o impacto do curso, foi realizada análise bivariada da variável-desfecho 'ter melhora' com as de exposição, aplicando-se os testes estatísticos específicos segundo o tipo da variável em questão, com nível de significância de $10 \%$. Se binária, aplicamos o teste qui-quadrado e a razão de chances; se contínua, o teste $\mathrm{T}$ de diferença de média. Em seguida, aplicou-se regressão logística para identificar o OR ajustado (ORAJ), de maneira a expressar o efeito ajustado do desfecho 'ter melhora', quando controlado por outras variáveis de exposição. O método utilizado foi o stepwise backward, ao nível de significância de 10\%, com probabilidade de entrada de 0,20 e de saída de 0,10. A próxima seção traz as respectivas proporções de classificação total do modelo logístico (PCT-Overall), ORAJ e respectivos intervalos de confiança de $95 \%$ (IC95\%).

\section{Resultados}

Na Tabela 1, é apresentado o perfil de 1.561 egressos do curso obtido na base Simios, segundo as ofertas do curso. 
Tabela 1

Perfil dos egressos do curso a distância em envelhecimento e saúde da pessoa idosa da Escola Nacional de Saúde Pública Sergio Arouca/Fundação Oswaldo Cruz, segundo variáveis selecionadas - Brasil, 2009-2013

\begin{tabular}{|c|c|c|c|c|}
\hline Perfil de egressos & $\begin{array}{c}\text { Oferta A } \\
2009-2010 \\
(n=193)\end{array}$ & $\begin{array}{c}\text { Oferta B } \\
2011-2013 \\
(n=1.363)\end{array}$ & $\begin{array}{l}\text { Total geral } \\
(n=1.561)\end{array}$ & $\begin{array}{c}\text { P_valor } \\
\left(\mathbf{x}^{2}\right)\end{array}$ \\
\hline Mulheres & $178(92,2 \%)$ & $1.236(90,4 \%)$ & $1.414(90,6 \%)$ & 0,403 \\
\hline \multicolumn{5}{|l|}{ Idade (faixa etária) } \\
\hline Menor que 35 anos & $32(16,6 \%)$ & $435(31,8 \%)$ & $467(29,9 \%)$ & \multirow{3}{*}{0,000} \\
\hline 35 a 50 anos & $96(49,7 \%)$ & $602(44,0 \%)$ & $698(44,7 \%)$ & \\
\hline Maior que 50 anos & $65(33,7 \%)$ & $331(24,2 \%)$ & $396(25,4 \%)$ & \\
\hline \multicolumn{5}{|l|}{ Profissão dos egressos } \\
\hline Assistente social & $16(8,3 \%)$ & $126(9,2 \%)$ & $142(9,1 \%)$ & \multirow{6}{*}{0,308} \\
\hline Enfermeiro(a) & $77(39,9 \%)$ & $539(39,4 \%)$ & $616(39,5 \%)$ & \\
\hline Fisioterapeuta & $17(8,8 \%)$ & $188(13,7 \%)$ & $205(13,1 \%)$ & \\
\hline Médico(a) & $24(12,4 \%)$ & $122(8,9 \%)$ & $146(9,4 \%)$ & \\
\hline Psicólogo(a) & $14(7,3 \%)$ & $105(7,7 \%)$ & $119(7,6 \%)$ & \\
\hline Outros & $45(23,3 \%)$ & $288(21,1 \%)$ & $333(21,3 \%)$ & \\
\hline \multicolumn{5}{|c|}{ Porte populacional do município (habitantes) } \\
\hline Até 50.000 & $13(6,7 \%)$ & $327(23,9 \%)$ & $340(21,8 \%)$ & \multirow{3}{*}{0,000} \\
\hline 50.001 a 100.000 & $5(2,6 \%)$ & $117(8,6 \%)$ & $122(7,8 \%)$ & \\
\hline Acima de 100.000 & $175(90,7 \%)$ & $924(67,5 \%)$ & 1.099 (70,4\%) & \\
\hline \multicolumn{5}{|l|}{ Região do país } \\
\hline Norte & $56(29 \%)$ & $0(0 \%)$ & $56(3,6 \%)$ & \multirow{5}{*}{0,000} \\
\hline Nordeste & $137(71 \%)$ & $0(0 \%)$ & $137(8,8 \%)$ & \\
\hline Sudeste & $0(0 \%)$ & 989 (72,3\%) & $989(63,4 \%)$ & \\
\hline Sul & $0(0 \%)$ & $260(19 \%)$ & $260(16,7 \%)$ & \\
\hline Centro-Oeste & $0(0 \%)$ & $119(8,7 \%)$ & $119(7,6 \%)$ & \\
\hline
\end{tabular}

Fonte: As autoras.

Houve importante predominância do sexo feminino em ambas as ofertas do curso, respectivamente $92,2 \%$ e $90,4 \%$ da clientela $(p=0,403)$. Similarmente, houve grande predominância de profissionais de enfermagem, cerca de $40 \%$, também sem diferença significativa entre as ofertas $(\mathrm{p}=0,308)$. A média de idade dos egressos das duas ofertas (A e B) variou entre 44,18 e 41,67 ( $p=0,032$ ) e média de 16,79 e 15,54 para cada oferta com relação ao tempo de formados $(p=0,294)$.

A despeito do predomínio de profissionais com idade entre 35 e 50 anos em ambas as ofertas $(49,7 \%$ e $44,0 \% ; p=0,000)$, a oferta B atraiu profissionais mais jovens (31,8\% com menos de 35 anos), identificando-se uma chance 1,6 vez maior de egressos com até 50 anos nessa oferta.

Observou-se diferença estatisticamente significativa na origem dos profissionais quanto ao porte populacional dos municípios. Houve predomínio de egressos provenientes de municípios com mais de 100 mil habitantes em 
ambas as ofertas (90,7\% na A e 67,5\% na B), embora tenha-se evidenciado na oferta $\mathrm{B}$ a presença três vezes maior de pessoas advindas de municípios com menos de 50 mil habitantes (6,7\% na oferta A e 23,9\% na oferta $B)$.

\section{Sobre os respondentes}

Na Tabela 2 é apresentado o perfil dos 414 respondentes. De forma convergente com o perfil geral dos egressos do curso, houve importante predominância do sexo feminino tanto na oferta A quanto na B, respectivamente $86,8 \%$ e $88,9 \%$. Da mesma forma, tem-se predominância de profissionais de enfermagem, cerca de $38 \%$, assim como de profissionais com idade entre 35 e 50 anos em ambas as ofertas (41,8\% e 43,3\%), sem diferença significativa entre elas.

\section{Tabela 2}

Perfil dos egressos respondentes do curso a distância em envelhecimento e saúde da pessoa idosa da Escola Nacional de Saúde Pública Sergio Arouca/Fundação Oswaldo Cruz, segundo variáveis selecionadas - Brasil, 2015

\begin{tabular}{|c|c|c|c|c|}
\hline $\begin{array}{l}\text { Perfil dos } \\
\text { respondentes }\end{array}$ & $\begin{array}{c}\text { Oferta A } \\
2009-2010 \\
(n=91)\end{array}$ & $\begin{array}{c}\text { Oferta B } \\
2011-2013 \\
(n=323)\end{array}$ & $\begin{array}{c}\text { Total geral } \\
(n=414)\end{array}$ & $\begin{array}{c}\text { P_valor } \\
\left(\mathbf{x}^{2}\right)\end{array}$ \\
\hline Mulheres & $79(86,8 \%)$ & $287(88,9 \%)$ & $366(88,4)$ & 0,591 \\
\hline \multicolumn{5}{|l|}{ Idade (faixa etária) } \\
\hline Menor que 35 anos & $24(26,4 \%)$ & $109(33,7 \%)$ & $133(32,1 \%)$ & \multirow{3}{*}{0,171} \\
\hline 35 a 50 anos & $38(41,8 \%)$ & $140(43,3 \%)$ & $178(43,0 \%)$ & \\
\hline Maior que 50 anos & $29(31,9 \%)$ & $74(22,9 \%)$ & $103(24,9 \%)$ & \\
\hline \multicolumn{5}{|l|}{ Profissão dos egressos } \\
\hline Assistente social & $12(13,2 \%)$ & $30(9,3 \%)$ & $42(10,1 \%)$ & \multirow{6}{*}{0,298} \\
\hline Enfermeiro(a) & $38(41,8 \%)$ & $120(37,2 \%)$ & $158(38,2 \%)$ & \\
\hline Fisioterapeuta & $9(9,9 \%)$ & $53(16,4 \%)$ & $62(15 \%)$ & \\
\hline Médico(a) & $10(11 \%)$ & $22(6,8 \%)$ & $32(7,7 \%)$ & \\
\hline Psicólogo(a) & $6(6,6 \%)$ & $30(9,3 \%)$ & $36(8,7 \%)$ & \\
\hline Outros & $16(17,6 \%)$ & $68(21,1 \%)$ & $84(20,3 \%)$ & \\
\hline \multicolumn{5}{|c|}{ Porte populacional do município (habitantes) } \\
\hline Até 50.000 & $19(20,9 \%)$ & $83(25,7 \%)$ & $102(24,6)$ & \multirow{3}{*}{0,201} \\
\hline 50.001 a 100.000 & $4(4,4 \%)$ & $28(8,7 \%)$ & $32(7,7 \%)$ & \\
\hline Acima de 100.000 & $68(74,7 \%)$ & $212(65,6 \%)$ & $280(67,6 \%)$ & \\
\hline \multicolumn{5}{|l|}{ Região do país } \\
\hline Norte & $14(15,4 \%)$ & $2(0,6 \%)$ & $16(3,9 \%)$ & \multirow{5}{*}{0,000} \\
\hline Nordeste & $26(28,6 \%)$ & $11(3,4 \%)$ & $37(8,9 \%)$ & \\
\hline Sudeste & $30(33 \%)$ & $204(63,2 \%)$ & $234(56,5 \%)$ & \\
\hline Sul & $15(15,6 \%)$ & $73(22,6 \%)$ & $88(21,3 \%)$ & \\
\hline Centro-Oeste & $6(6,6 \%)$ & $33(10,2 \%)$ & $39(9,4 \%)$ & \\
\hline
\end{tabular}

Fonte: As autoras. 
Manteve-se o predomínio de egressos provenientes de municípios com mais de 100 mil habitantes em ambas as ofertas (74,7\% na A e $65,6 \%$ na B).

\section{Efetividade do curso}

Na Tabela 3, apresenta-se a declaração dos entrevistados quanto à realização das atividades previstas para atendimento à população idosa na atenção básica, de acordo com as diretrizes da PNSPI e do Pacto pela Vida, em dois momentos distintos: antes do início e depois do término do curso. Encontraram-se diferenças significativas na proporção de egressos que não realizavam as atividades e passaram a realizá-las após o curso. Esse comportamento foi verificado para todas as atividades investigadas, com a proporção de implementação melhorando de 1,3 a 2,6 vezes, e as atividades 'uso da caderneta', 'aplicação de testes' e 'uso dos dados dos sistemas de informação em saúde' tiveram melhora de pelo menos duas vezes mais, quando comparados os dois períodos. Por exemplo, a atividade 'uso dos dados dos sistemas de informação em saúde' mudou de $25 \%$ para cerca de $60 \%$ de egressos passando a realizá-la.

\section{Tabela 3}

Desenvolvimento das atividades relacionadas à saúde do idoso antes e depois do curso e razão de proporção - Brasil, 2015

\begin{tabular}{|c|c|c|c|c|c|c|}
\hline \multirow[t]{2}{*}{ Atividades implementadas } & \multicolumn{2}{|c|}{$\begin{array}{c}\text { Antes do } \\
\text { início do curso } \\
\% \text { coluna }\end{array}$} & \multicolumn{2}{|c|}{$\begin{array}{c}\text { Depois do } \\
\text { término do curso } \\
\% \text { coluna }\end{array}$} & \multirow[t]{2}{*}{ P_valor ${ }^{1}$} & \multirow[t]{2}{*}{$\begin{array}{l}\text { Razão } \\
(2) /(1)\end{array}$} \\
\hline & $\mathbf{N}$ & (1) & $\mathbf{N}$ & (2) & & \\
\hline Uso da caderneta & 89 & $21,5 \%$ & 174 & $42,0 \%$ & 0,000 & 2,0 \\
\hline Aplicação de testes & 106 & $25,6 \%$ & 273 & $65,9 \%$ & 0,000 & 2,6 \\
\hline Educação em saúde/prevenção de quedas & 223 & $53,9 \%$ & 350 & $84,5 \%$ & 0,000 & 1,6 \\
\hline Busca ativa de idosos & 133 & $32,1 \%$ & 245 & $59,2 \%$ & 0,000 & 1,8 \\
\hline Atividades de estímulo ao envelhecimento ativo & 291 & $70,3 \%$ & 371 & $89,6 \%$ & 0,000 & 1,3 \\
\hline Atividades de promoção do autocuidado & 267 & $64,5 \%$ & 351 & $84,8 \%$ & 0,000 & 1,3 \\
\hline $\begin{array}{l}\text { Uso dos dados dos sistemas de informação } \\
\text { em saúde }\end{array}$ & 103 & $24,9 \%$ & 246 & $59,4 \%$ & 0,000 & 2,4 \\
\hline $\begin{array}{l}\text { Identificação de instituições/organizações } \\
\text { existentes na comunidade }\end{array}$ & 211 & $51,0 \%$ & 365 & $88,2 \%$ & 0,000 & 1,7 \\
\hline Informações para a comunidade & 286 & $69,1 \%$ & 386 & $93,2 \%$ & 0,000 & 1,3 \\
\hline Desenvolvimento de estratégias paliativas & 177 & $42,8 \%$ & 279 & $67,4 \%$ & 0,000 & 1,6 \\
\hline
\end{tabular}


Na Tabela 4, apresentam-se os resultados dos testes de associação entre cada uma das variáveis de exposição consideradas e o desfecho 'ter melhora'. Encontrou-se associação significativa ( $\mathrm{p}<10 \%)$ para: 'idade até 50 anos', 'trabalhar em municípios com porte populacional até 50 mil habitantes', 'ser enfermeiro ou médico', 'trabalhar nesta área (saúde do idoso) há até 5 anos' e 'ter conseguido implementar na prática o curso'.

Nenhum dos aspectos referidos como facilidade/dificuldade para implantação ou aprimoramento apresentou significância estatística com o desfecho 'ter melhora'. Cabe destacar, porém, aqueles identificados como facilitadores pelos egressos que melhoraram seu desempenho em pelo menos cinco das atividades preconizadas: 'gestão local de saúde', 'estrutura física do local' e 'infraestrutura de apoio'; e como aspectos que dificultavam a implantação das atividades: 'acesso da população ao serviço', 'quantidade de profissionais', 'quantidade de profissionais capacitados' e 'rede de serviço de saúde'.

\section{Tabela 4}

Variáveis investigadas segundo melhora ou não melhora no desempenho de pelo menos cinco atividades relacionadas à saúde do idoso - Brasil, 2015

\begin{tabular}{|c|c|c|c|c|}
\hline \multirow{2}{*}{ Variáveis testadas (exposição) } & \multicolumn{4}{|c|}{ Melhora em pelo menos cinco atividades ${ }^{1}$} \\
\hline & $\operatorname{Sim}(\%)$ & Não (\%) & Total (\%) & \\
\hline Total de entrevistados & $\begin{array}{c}107 \\
(100 \%)\end{array}$ & $\begin{array}{c}307 \\
(100 \%)\end{array}$ & $\begin{array}{c}414 \\
(100 \%)\end{array}$ & P_valor \\
\hline Mulheres & $87,9 \%$ & $88,6 \%$ & $88,4 \%$ & 0,835 \\
\hline Idade até 50 anos & $81,3 \%$ & $73,0 \%$ & $75,1 \%$ & 0,086 \\
\hline $\begin{array}{l}\text { Trabalha em município com porte populacional até } 50 \text { mil } \\
\text { habitantes }\end{array}$ & $30,8 \%$ & $22,5 \%$ & $24,6 \%$ & 0,084 \\
\hline Egresso da $1^{\text {a }}$ oferta do curso & $23,4 \%$ & $21,5 \%$ & $22,0 \%$ & 0,688 \\
\hline É enfermeiro ou médico & $55,1 \%$ & $42,7 \%$ & $45,9 \%$ & 0,038 \\
\hline Está cursando/cursou outro curso na mesma área & $29,0 \%$ & $28,3 \%$ & $28,5 \%$ & 00,901 \\
\hline Não desenvolve a mesma função da época do curso & $32,7 \%$ & $28,0 \%$ & $29,2 \%$ & 0,358 \\
\hline Trabalha com saúde do idoso há até cinco anos & $39,3 \%$ & $28,0 \%$ & $30,9 \%$ & 0,030 \\
\hline $\begin{array}{l}\text { Está satisfeito ou muito satisfeito em trabalhar com saúde } \\
\text { do idoso }\end{array}$ & $78,5 \%$ & $72,6 \%$ & $74,2 \%$ & 0,233 \\
\hline $\begin{array}{l}\text { Não continua trabalhando no mesmo município da época } \\
\text { do curso }\end{array}$ & $21,5 \%$ & $20,5 \%$ & $20,8 \%$ & 0,831 \\
\hline
\end{tabular}


Continuação - Tabela 4

Variáveis investigadas segundo melhora ou não melhora no desempenho de pelo menos cinco atividades relacionadas à saúde do idoso - Brasil, 2015

\begin{tabular}{|c|c|c|c|c|}
\hline \multirow{2}{*}{ Variáveis testadas (exposição) } & \multicolumn{4}{|c|}{ Melhora em pelo menos cinco atividades' } \\
\hline & $\operatorname{Sim}(\%)$ & Não (\%) & Total (\%) & \\
\hline Total de entrevistados & $\begin{array}{c}107 \\
(100 \%)\end{array}$ & $\begin{array}{c}307 \\
(100 \%)\end{array}$ & $\begin{array}{c}414 \\
(100 \%)\end{array}$ & P_valor \\
\hline $\begin{array}{l}\text { Desenvolve como atividade principal ações assistenciais/ } \\
\text { educativas }\end{array}$ & $52,3 \%$ & $47,9 \%$ & $49,0 \%$ & 0,427 \\
\hline $\begin{array}{l}\text { Acha que o curso foi fortemente relacionado ao trabalho } \\
\text { na saúde do idoso }\end{array}$ & $75,7 \%$ & $71,7 \%$ & $72,7 \%$ & 0,419 \\
\hline $\begin{array}{l}\text { Acha que formação recebida no curso é compatível com a } \\
\text { necessidade de conhecimento para a prática atual }\end{array}$ & $76,6 \%$ & $69,7 \%$ & $71,5 \%$ & 0,139 \\
\hline $\begin{array}{l}\text { Conseguiu implementação prática do curso saúde do } \\
\text { idoso }\end{array}$ & $40,2 \%$ & $26,4 \%$ & $30,0 \%$ & 0,007 \\
\hline \multicolumn{5}{|c|}{$\begin{array}{l}\text { Aspectos referidos como facilidade/dificuldade para implantação } \\
\text { ou aprimoramento das atividades da saúde do idoso }\end{array}$} \\
\hline Gestão local de saúde (facilidade) & $52,3 \%$ & $48,5 \%$ & $49,5 \%$ & 0,498 \\
\hline Estrutura física do local (facilidade) & $47,7 \%$ & $38,8 \%$ & $41,1 \%$ & 0,107 \\
\hline Infraestrutura de apoio (facilidade) & $23,4 \%$ & $19,5 \%$ & $20,5 \%$ & 0,400 \\
\hline Acesso da população ao serviço (dificuldade) & $36,4 \%$ & $33,6 \%$ & $34,3 \%$ & 0,587 \\
\hline Quantidade adequada de profissionais (dificuldade) & $84,1 \%$ & $82,1 \%$ & $82,6 \%$ & 0,634 \\
\hline $\begin{array}{l}\text { Quantidade suficiente de profissionais capacitados } \\
\text { (dificuldade) }\end{array}$ & $90,7 \%$ & $87,0 \%$ & $87,9 \%$ & 0,314 \\
\hline Rede de serviço de saúde (dificuldade) & $72,0 \%$ & $68,4 \%$ & $69,3 \%$ & 0,492 \\
\hline
\end{tabular}

\section{Fonte: As autoras}

Nota: 'Atividades: uso da caderneta; aplicação de testes; educação em saúde/prevenção de quedas; busca ativa de idosos atividades de estímulo ao envelhecimento ativo; atividades de promoção do autocuidado; uso dos dados dos sistemas de informação em saúde; identificação de instituições/organizações existentes na comunidade; informações para a comunidade; desenvolvimento de estratégias paliativas.

Na abordagem multivariada (Tabela 5), as variáveis que tiveram maior influência significativa na melhora do desempenho $(\mathrm{p}<10 \%)$ foram: profissão (ser assistente social, fisioterapeuta ou psicólogo [OR=1,834; $\mathrm{p}=0,097] \mathrm{e}$ ser médico ou enfermeiro [OR=2,629; $\mathrm{p}=0,005]$, em comparação com outras profissões); tempo que trabalha com saúde do idoso (SI) tendo como referência mais de cinco anos de atuação com essa clientela ( $O R=1,776 ; p=0,018)$; e a implementação prática do curso quando comparada a sua não implementação $(\mathrm{OR}=2,010 ; \mathrm{p}=0,004)$. 
Tabela 5

Regressão logística com método stepwise backward para o desfecho melhora no desempenho dos profissionais em pelo menos cinco atividades ${ }^{1}$ - Brasil, 2015

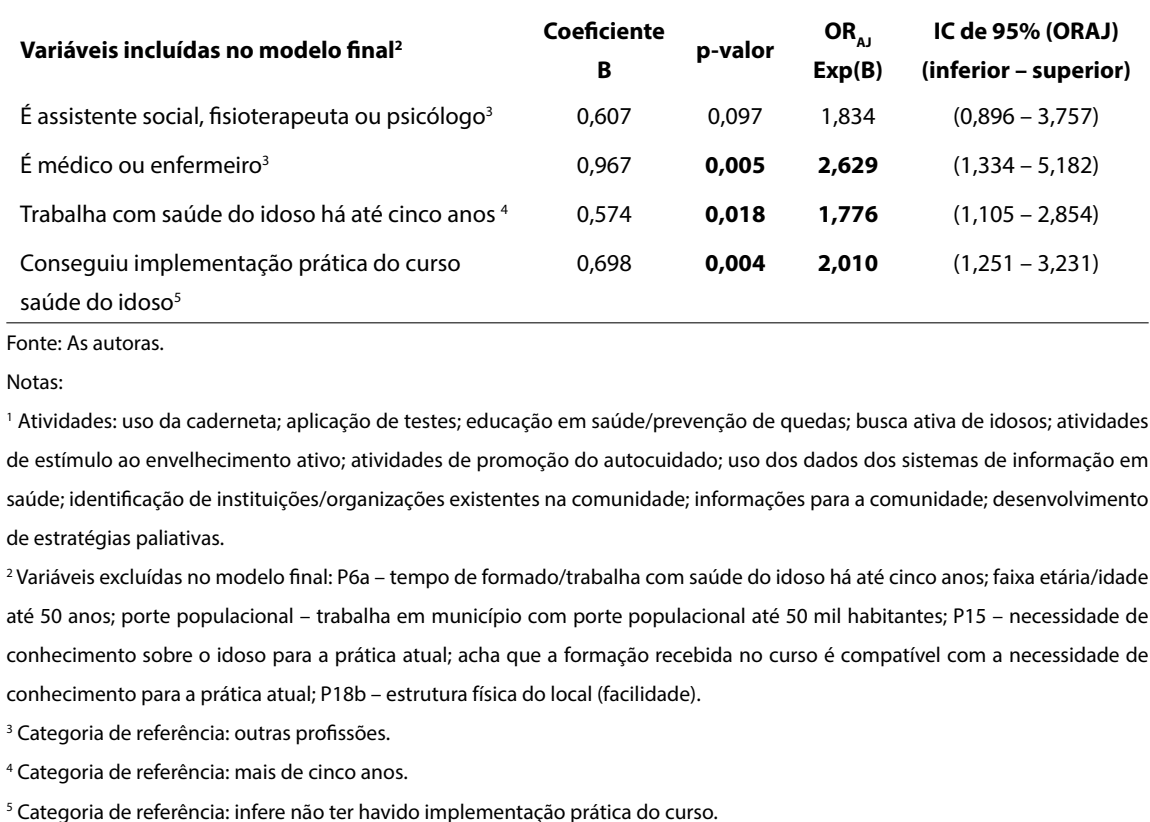

Esse modelo é o que melhor explica a probabilidade de 'ter melhora', com $61 \%$ de probabilidade de classificação total, estimando aqueles que apresentavam melhora uma proporção de classificação de $55,7 \%$; e entre aqueles que não apresentavam, proporção de $62,9 \%$ de acerto na classificação.

\section{Discussão}

Os egressos foram predominantemente mulheres, com maior presença de profissionais da enfermagem, sendo mais jovens na oferta B (regiões Sul/Sudeste/ Centro-Oeste). Os municípios com mais egressos do curso são aqueles com população superior a 100 mil habitantes, havendo um incremento de cerca de 3,5 vezes dos municípios com população com menos de 50 mil habitantes que tiveram egressos do curso na oferta B. Esse mesmo perfil foi identificado na pesquisa de campo. Pode-se dizer que o grupo de 414 respondentes representa adequadamente os 1.561 egressos das ofertas avaliadas do curso.

Em que pese a estratégia do ensino a distância ter como objetivo ampliar o alcance da educação permanente, vantagem com especial importância no Brasil em virtude de seu grande território e número de municípios, observouse uma baixa captação relativa de profissionais de municípios pequenos - o 
que pode ter ocorrido pelas dificuldades nas condições tecnológicas para participação no curso, como a baixa oferta de internet nesses locais.

Estudos com egressos de cursos na área da saúde são escassos no país, e de fato não foram encontrados trabalhos abordando esse mesmo público-alvo, egressos de curso EAD de aperfeiçoamento em saúde. A literatura (Craveiro et al., 2015; Teixeira, 2013) aponta a existência de uma lacuna de estudos sobre o posicionamento dos profissionais da saúde, com referência tanto a sua prática quanto ao sistema ou à própria política de saúde.

Em relação à caracterização do perfil de egressos, estudo que abordou cursos de saúde coletiva de algumas universidades do país também encontrou maioria feminina e indicação de preferência em residir nas capitais, isto é, em municípios com população acima de 100 mil habitantes (Castellanos et al., 2013). Estudos com egressos de cursos de mestrado e doutorado na área da saúde também apresentaram prevalência feminina (Gomes e Goldenberg, 2010; Hortale et al., 2010). Em nossos achados, a predominância de mulheres está possivelmente associada à profissão de enfermagem, essencialmente feminina. A noção de cuidado, ação identificada como feminina (Lopes e Leal, 2005), é usada como argumento da predominância das mulheres na atuação no campo da enfermagem.

A diferença observada quanto à região de origem dos egressos, assim como dos respondentes, é apenas descritiva, uma vez que cada oferta foi especificamente direcionada a grupos de regiões (oferta A para N/NE e oferta B para SE/S/CO).

Para o amplo e bom uso dos serviços de saúde é preciso haver, antes de mais nada, vontade política, planejamento e comprometimento com a população. O serviço de saúde precisa estar estruturado para atender às necessidades inerentes ao idoso, de modo a possibilitar seu acesso e respeitar suas limitações.

Piccini e colaboradores (2006), ao compararem a efetividade da utilização dos serviços da atenção básica em saúde (ABS) pelos idosos no modelo tradicional e no Programa Saúde da Família (PSF) nas regiões Nordeste e Sul, encontrou alta proporção de inadequação no acesso a pessoas com deficiência, variando de $68 \%$ a $54 \%$. O estudo ainda relata outros problemas, como a ausência de cadeiras na sala de espera, a indisponibilidade de cadeiras de rodas, a falta de protocolo para cuidados domiciliares e a ausência de capacitação dos trabalhadores para lidar com os casos de hipertensão e diabetes. Estrutura física do local e infraestrutura de apoio foram apontados como fatores facilitadores para implantação ou aprimoramento das atividades da saúde do idoso em nosso estudo. Assim, é razoável supor que a iniciativa de apoiar a educação permanente de profissionais esteja associada a um conjunto de esforços positivos de gestão que inclua a preocupação com a estrutura dos serviços, em que pese abordarmos apenas a perspectiva dos profissionais na pesquisa que deu origem a este artigo. 
Entretanto, convergentemente com Piccini e colaboradores (2006), os egressos relataram a ausência de profissionais capacitados como sendo um fator de dificuldade na implementação das atividades preconizadas pela PNSPI. O número reduzido de profissionais que tenham realizado capacitação voltada para o cuidado ao idoso e a quantidade reduzida de profissionais disponíveis para atuar nas necessidades dessa população podem interferir tanto na qualidade quanto na baixa utilização dessa clientela específica dos serviços ofertados - o que contribui para a perda da efetividade do cuidado prescrito na PNSPI e no Estatuto do Idoso (Piccini et al., 2006).

Assim, a ausência de profissionais especialistas ou mesmo de serviços especializados voltados para idosos colocam em xeque a efetividade das políticas propostas para os cuidados a esse público. Os profissionais que trabalham com essa clientela precisam estar capacitados para atender necessidades específicas, de forma integrada com as outras práticas da rede de cuidado.

A adequada abordagem da saúde do idoso traz o desafio de dar conta de uma ampla gama de questões tanto multiprofissionais quanto intersetoriais, que precisarão atuar juntas na conjunção de diversas dimensões como a social, a psicológica e a física. Fica assim claro o desafio de um curso que se pretenda estratégia para superação dos desafios. Há, ademais, que se considerar que os egressos variam em número dentro de um mesmo município e não necessariamente conseguirão conjugar seus esforços para o alcance de resultados. A própria definição de resultado relevante poderá variar a depender da acumulação e trajetória de cada profissional. Ainda que não pretenda trazer respostas prontas, a teoria construcionista crítica (Sousa Filho, 2012) lança luzes nesse cenário, aguçando o olhar aos obstáculos a serem enfrentados.

As variáveis com relação à profissão (ser assistente social, fisioterapeuta ou psicólogo e ser médico ou enfermeiro, em comparação a outras profissões), tempo que trabalha com SI, tendo como referência mais de cinco anos de atuação com essa clientela, e a implementação prática do curso em comparação com a sua não implementação são as que melhor explicam (61\%) a probabilidade de 'ter melhora' em pelo menos cinco das dez atividades preconizadas.

Estudo anterior sobre a mesma população de egressos do curso relata que os profissionais que não compõem a equipe mínima da ESF (ou seja, não são nem médicos nem enfermeiros) referem que o curso é muito voltado para os profissionais da equipe mínima, não contemplando as outras profissões, que são complementares e fundamentais ao trabalho em equipe (Torres, 2012).

Enquanto os profissionais que não compõem a equipe mínima da ESF relataram que o curso não faz diferença para o trabalho deles, de forma distinta os médico/enfermeiros apresentaram a maior melhora. No entanto, pudemos evidenciar por meio da abordagem multivariada a melhora no desenvolvimento das atividades preconizadas também para assistentes sociais, fisioterapeutas e 
psicólogos, quando comparados às outras categorias profissionais, excluídos os médicos/enfermeiros.

É de nosso conhecimento que o curso em estudo passou por processo de atualização para as ofertas seguintes às que aqui foram analisadas, a fim de minimizar esse distanciamento relatado pelos profissionais considerados como complementares à equipe da ESF. No entanto, não temos como avaliar se esse distanciamento foi solucionado.

No que tange às limitações e forças do estudo aqui apresentado, a utilização de bases secundárias (Sistema Simios) trouxe como limitação a qualidade/ abrangência dos dados a serem analisados. Por se tratar de um sistema em que o preenchimento é feito pelo próprio aluno, houve perda de informações importantes para a análise, como sexo, profissão e município de atuação.

Outro ponto diz respeito à utilização de questionário eletrônico. Ainda que se reconheça a importância de se ouvir o sujeito do estudo, isso não foi possível por se tratar de uma pesquisa de âmbito nacional, de maneira que, devido a constrangimentos de tempo e recursos, optamos pelo questionário eletrônico anônimo de autopreenchimento. Adicionalmente, o formato de questionário utilizado (eletrônico) é reconhecido por sua taxa de não resposta, ainda que se aponte que isso não afeta a validade interna dos resultados, mas pode comprometer seu poder quanto à validade externa, já que a representatividade das respostas é apontada como fator mais importante, a exemplo das pesquisas eleitorais (Cook, Heath e Thompson, 2000). As desvantagens relacionadas à falta de acesso à internet de parte da população, assim como a falta de familiaridade com equipamentos eletrônicos, não se aplicam ao universo aqui estudado, já que tais condições eram requisito para se ter realizado o curso em análise. A realização de teste-piloto buscou minimizar os problemas relacionados à interpretação e à compreensão das perguntas na ausência de entrevistador. Por sua vez, os questionários eletrônicos têm como vantagens a rapidez na coleta das informações, a viabilidade de aplicação a um grande grupo em razão da facilidade de acesso ao instrumento e a possibilidade de se montar um banco de dados por meio das informações obtidas, de modo a tornar a análise rápida e precisa.

Perdas adicionais podem ter ocorrido em virtude de informações defasadas, em consequência de os alunos não terem o costume de atualizar os dados pessoais cadastrados na instituição depois da conclusão do curso. Por se tratar de estudo transversal, não é possível inferir acerca de relação de causalidade.

\section{Considerações finais}

A avaliação de intervenções públicas, incluindo a de egressos de cursos realizados com recursos governamentais, se faz necessária, uma vez que a educação 
permanente compõe o conjunto de estratégias de muitas políticas públicas, incluindo a PNSPI. O estudo aqui apresentado fornece uma peça ao mosaico, devendo ser complementado com abordagens que contemplem os efeitos concretos na população-alvo e na promoção de mudanças nos processos de trabalho e na rede de atendimento do SUS.

A Fiocruz, do ponto de vista acadêmico e político, ocupa destacada posição na formação profissional e na formulação de diretrizes para o desenvolvimento científico e tecnológico no campo da saúde e pode contribuir para esse processo de análise do impacto de seus cursos.

Formação profissional, tempo de trabalho com saúde do idoso e implementação prática do aprendizado obtido no curso foram os aspectos que melhor explicaram a melhora nas atividades do PNSPI. Nossos achados reforçam a importância da educação permanente como ferramenta para formação/capacitação dos profissionais que atuam no SUS. Embora ainda confundida com educação continuada, a educação permanente vem se desenvolvendo em todas as regiões do país. $\mathrm{O}$ uso das tecnologias de informação na EAD reforçam e potencializam a educação permanente como estratégia para disseminação do processo educativo.

Concluímos que a educação permanente é uma ferramenta importante para implementação de políticas em saúde. Dessa forma, o curso mostrou-se como estratégia relevante na implementação de atividades da PNSPI.

As lacunas identificadas na literatura brasileira quanto à avaliação de cursos de educação profissional apontam a necessidade de estudos futuros. Alguns aspectos relevantes a serem abordados: os efeitos na população-alvo e a magnitude das possíveis mudanças causadas na oferta dos serviços à população idosa. 


\section{Colaboradoras}

Kellem Raquel Brandão de Oliveira Torres, Vera Lucia Luiza e Mônica Rodrigues Campos participaram de todas as etapas da construção do artigo, desde a concepção, o delineamento e a análise até a redação final.

\section{Agradecimentos}

Aos 414 profissionais egressos do curso em estudo por participarem da pesquisa, possibilitando sua realização, e a todos da EAD/Ensp/Fiocruz, em especial: a coordenadora da EAD/Ensp, Lucia Dupret; a coordenadora do curso, Angela Castilho, e toda a sua equipe de orientadores; as amigas Suely Guimarães, Cleide Figueiredo, Rosa Rocha, Maria Angélica, Milta Torrez e Simone Agadir; aos profissionais da informática; e ao casal Lídia Bandeira Coelho e Diogo Galvão.

Resumen Este artículo tiene como objetivo analizar la efectividad del curso de perfeccionamiento en envejecimiento y salud de la tercera edad para la implementación de la Política Nacional de Salud para la Vejez, teniendo en cuenta la percepción de profesionales egresos. Un cuestionario electrónico de respuesta automática fue implementado de Julio a Octubre 2015, completado por 414 de los 1,561 egresos. La variable para medir el impacto del curso fue la mejora en el desempeño de los egresos en al menos 5 de las 10 actividades recomendadas en la Política Nacional de Salud para la Vejez. Hubo mejora en la realización de todas las actividades, variando de 1.3 a 2.6 veces pre y pos curso. Formación profesional, tiempo de trabajo con salud de majores, y implementación práctica del aprendizaje obtenido en el curso fueron los aspectos que mejor explicaron la mejora en las actividades de la política (por modelo logístico de regresión). El curso se mostró una estrategia efectiva para la implementación de las actividades de la política asi como el potencial de la educación continuada en fortalecer políticas públicas.

Palabras-clave salud de la tercera edad; educación a distancia; educación continua. 


\section{Notas}

${ }^{1}$ Fundação Oswaldo Cruz, Escola Nacional de Saúde Pública Sergio Arouca, Departamento de Política de Medicamentos e Assistência Farmacêutica, Rio de Janeiro, Rio de Janeiro, Brasil. $<$ kellemraquelbo@gmail.com>

Correspondência: Fundação Oswaldo Cruz, Escola Nacional de Saúde Pública Sergio Arouca, Rua Leopoldo Bulhões, 1.480, Prédio Joaquim Cardoso de Melo, CEP 21041210, Manguinhos, Rio de Janeiro, Rio de Janeiro, Brasil.

${ }^{2}$ Fundação Oswaldo Cruz, Escola Nacional de Saúde Pública Sergio Arouca, Departamento de Política de Medicamentos e Assistência Farmacêutica, Rio de Janeiro, Rio de Janeiro, Brasil. $<$ vera@ensp.fiocruz.br>

${ }^{3}$ Fundação Oswaldo Cruz, Escola Nacional de Saúde Pública Sergio Arouca, Departamento de Ciências Sociais, Rio de Janeiro, Rio de Janeiro, Brasil.

$<$ monicarodriguescampos@gmail.com> 


\section{Referências}

ABREU JR., Nelson. Sistema(s) de avaliação da educação superior brasileira. Cadernos Cedes [online], Campinas, v. 29, n. 78, p. 257-269, 2009.

BELLO, Isabel M.; RODRIGUES, Ana C. C. Educação a distância, formação inicial de professores, desenvolvimento profissional e pessoal: uma combinação possível? Cadernos de Educação, Pelotas, n. 41, p. 208-229, jan. 2012 .

BRASIL. Ministério da Saúde. Norma operacional básica do Sistema Único de Saúde/ SUS. Diário Oficial da República Federativa do Brasil, Poder Executivo, Brasília, DF, 6 nov. 1996. Seção 1, p. 48.

BRASIL. Ministério da Saúde. Redes estaduais de atenção à saúde do idoso: guia operacional e portarias relacionadas. Brasília, DF, 2002.

BRASIL. Ministério da Saúde. Secretaria de Gestão do Trabalho na Saúde. Departamento de Gestão da Educação na Saúde. Política de educação e desenvolvimento para o SUS: caminhos para a EPS - polos de educação permanente em saúde. Brasília, DF, 2004.

BRASIL. Ministério da Saúde. A educação permanente entra na roda: polos de educação permanente em saúde - conceitos e caminhos a percorrer. Brasília, DF, 2005.

BRASIL. Ministério da Saúde. Portaria n. 2.528, de 19 de outubro de 2006. Aprova a Política Nacional de Saúde da Pessoa Idosa. 2006. Disponível em: <http://www.saudeidoso.icict.fiocruz.br/pdf/PoliticaNacionaldeSaudedaPessoaIdosa.pdf $>$. Acesso em: 5 dez. 2015.

BRASIL. Ministério da Saúde. Portaria n. 1.996, de 20 de agosto de 2007. Dispõe sobre a implementação da Política Nacional de Educação Permanente em Saúde. Disponível em: <http:// www.saude.pb.gov.br/site/geab/portaria2007. pdf $>$. Acesso em: 5 dez. 2015.
BRASIL. Ministério da Saúde. Vigitel Brasil 2014. vigilância de fatores de risco e proteção para doenças crônicas por inquérito telefônico. Brasília: Ministério da Saúde, 2015. Acesso em: 5 dez. 2015.

BROCHADO, Marina R.; PHITON, Antônio J. C.; PEREIRA, Marcelo C. QFD instrumento de autoavaliação nas instituições de ensino superior. In: ENCONTRO NACIONAL DE ENGENHARIA DE PRODUÇÃO(ENEGEP), 26., 2006, Fortaleza. Anais eletrônicos... Fortaleza: Abepro, 2006. Disponível em: <http://www.abepro.org.br/biblioteca/ enegep2006_tr540363_8578.pdf>. Acesso em: 5 dez. 2015.

CASTELLANOS, Marcelo. E. P. et al. Estudantes de graduação em saúde coletiva: perfil sociodemográfico e motivações. Ciência \& Saúde Coletiva, Rio de Janeiro, v. 18, n. 2, p. 1.657-1.666, 2013.

CECCIM, Ricardo B. Educação permanente em saúde: descentralização e disseminação de capacidade pedagógica na saúde. Ciência \& Saúde Coletiva, Rio de Janeiro, v. 10, n. 4, p. 975-986, 2005.

CECCIM, Ricardo B.; FERLA, Alcindo A. Educação permanente em saúde. In: PEREIRA, Isabel B.; LIMA, Julio C. F. (orgs.). Dicionário da Educação Profissional em Saúde. 2. ed. Rio de Janeiro: Escola Politécnica de Saúde Joaquim Venâncio; Fundação Oswaldo Cruz 2009. Disponível em: <http://www.epsjv. fiocruz.br/dicionario/verbetes/edupersau. html>. Acesso em: 7 nov. 2014.

COIMBRA, Angela M. C. et al. Envelhecimento e saúde da pessoa idosa. Caderno do aluno: orientações para o curso. Rio de Janeiro: Escola Nacional de Saúde Pública Sergio Arouca; Fundação Oswaldo Cruz, 2008. 60 p.

COOK, Colleen.; HEATH, Fred.; THOMPSON, Russel. L. A meta-analysis of response rates in web-or internet-based surveys. Educational and Psychological Measurement, Texas, v. 60, n. 6, p. 821-836, dez. 2000. 
CORDEIRO, Célio O.; MOTTA, Regis R. A qualidade do ensino pela ótica do cliente. In: ENCONTRO NACIONAL DE ENGENHARIA DE PRODUÇÃO (ENEGEP), 28., Rio de Janeiro, 2008. Anais eletrônicos... Rio de Janeiro: Abepro, 2008. Disponível em: <http:// www.abepro.org.br/biblioteca/enegep2008_ TN_STO_070_502_12315.pdf $>$. Acesso em: 5 dez. 2015.

CRAVEIRO, Isabel M. R. et al. Desigualdades sociais, políticas de saúde e formação de médicos, enfermeiros e dentistas no Brasil e em Portugal. Ciência \& Saúde Coletiva, Rio de Janeiro, v. 20, n. 10, p. 2.985-2.998, out. 2015.

ESPARTEL, Lélis B. O uso da opinião dos egressos como ferramenta de avaliação de cursos: o caso de uma instituição de ensino superior catarinense. Revista Alcance [online], Itajaí, v. 16, n. 1, p. 102-114, jan.-abr. 2009. Disponível em: <http://siaiap32.univali.br/ seer/index.php/ra/article/view/1050/859>. Acesso em: 30 nov. 2014.

GADOTTI, Moacir. A Educação contra a Educação. 5. ed. Rio de Janeiro: Paz e Terra, 1992.

GOMES, Mara. H. A.; GOLDENBERG, Paulete. Retrato quase sem retoques dos egressos dos programas de pós-graduação em saúde coletiva, 1998-2007. Ciência \& Saúde Coletiva, Rio de Janeiro, v. 15, n. 4, p. 1.989-2.005, 2010.

GOMES, Victor. Envelhecimento demandará grandes investimentos em hospitais. Blog Economia de serviços: um espaço para debates. 2016. Disponível em: <http://economiadeservicos. com/2016/01/21/envelhecimento-demandaragrandes-investimentos-em-hospitais/> . Acesso em: 3 fev. 2016.

HARTZ, Zulmira. Do monitoramento do desempenho ao desempenho do monitoramento: novas oportunidades para a avaliação na gestão da vigilância em saúde. Ciência \& Saúde Coletiva, Rio de Janeiro, v. 18, n. 5, p. 1.221-1.222, 2013.

HORTALE, Virgínia. A. et al. Características e limites do mestrado profissional na área da saúde: estudo com egressos da Fundação Oswaldo Cruz. Ciência \& Saúde Coletiva, Rio de Janeiro, v. 15, n. 4, p. 2.051-2.058, 2010.

LEITÃO, Cleide. F. et al. Formação de tutores para o curso de formação pedagógica em educação profissional na área de saúde: enfermagem. Rio de Janeiro: Escola Nacional de Saúde Pública Sergio Arouca; Fundação Oswaldo Cruz, 2000.

LIMA, Josiane. V. C. et al. Continuing education in health as a pedagogical strategy to transform the practice: possibilities and limits. Trabalho, Educação e Saúde, Rio de Janeiro, v. 8 , n. 2, p. 207-227, 2010.

LOPES, Marta. J. M.; LEAL, Sandra. M. C. A feminização persistente na qualificação profissional da enfermagem brasileira. Cadernos Pagu, Campinas, v. 24, p. 105-125, jan.-jun. 2005.

LOUSADA, Ana. C. Z.; MARTINS, Gilberto. A. Egressos como fonte de informação à gestão dos cursos de ciências contábeis. Revista de Contabilidade e Finanças-USP, São Paulo, n. 37, p. 73-84, jan.-abr. 2005.

MALTA, Deborah. C. et al. Prevalência de fatores de risco e proteção para doenças crônicas não transmissíveis em adultos residentes em capitais brasileiras, 2013. Epidemiologia e Serviços de Saúde, Brasília, v. 24, n. 3, p. 373-387, jul.-set. 2015.

PERRENOUD, Philippe. Construindo competências: entrevista [set. 2000]. Entrevistadoras: Paola Gentile e Roberta Bencini. Disponível em: <http://www.unige.ch/fapse/SSE/teachers/ perrenoud/php_main/php_2000/2000_31. html>. Acesso em: nov. 2014.

PICCINI, Roberto X. et al. Necessidades de saúde comuns aos idosos: efetividade na oferta e utilização em atenção básica à saúde. Ciência \& Saúde Coletiva, Rio de Janeiro, v. 11, n. 3, p. 657-667, 2006.

RODRIGUES, Francisco. A aventura da qualidade. Boletim Técnico do Senai, São Paulo, maio-ago. 1994. 
SILVESTRE, Jorge. A.; COSTA NETO, Milton. M. Abordagem do idoso em programas de saúde da família. Cadernos de Saúde Pública, Rio de Janeiro, v. 19, n. 3, p. 839-847, maiojun. 2003.

SOUSA FILHO, Alípio. Por uma teoria construcionista crítica. Bagoas: Estudos Gays gêneros e sexualidades, Natal, v. 1, n. 1, p. 27-59, 2007.

TEIXEIRA, Carmen. F. S. Health professionals and workers in Brazil: where do we go from here? Ciência \& Saúde Coletiva, Rio de Janeiro, v. 18, n. 6, p. 1.535-1.536, 2013.

TORRES, Kellem R. B. O. Curso de aperfeiçoamento em envelhecimento e saúde da pessoa idosa
(EAD/Ensp/Fiocruz): uma avaliação de alunos e tutores. Pôster eletrônico. In: CONGRESSO BRASILEIRO DE SAÚDE COLETIVA, 10., Porto Alegre, 2012. Anais Saúde Coletiva. Porto Alegre: 2012. Disponível em: <http:// abrasco.org.br/saudecoletiva2012/>. Acesso em: 25 nov. 2014

VERAS, Renato. P.; CALDAS, Célia. P. Promovendo a saúde e a cidadania do idoso: o movimento das universidades da terceira idade. Ciência \& Saúde Coletiva, Rio de Janeiro, v. 9, n. 2, p. 423-432, 2004.

VIACAVA, Francisco. et al. Avaliação de desempenho de sistemas de saúde: um modelo de análise. Ciência \& Saúde Coletiva, Rio de Janeiro, v. 17, n. 4, p. 921-934, 2012.

Recebido em 25/05/2016.

Aprovado em 17/03/2017. 